\title{
On a 'time' reparametrization in relativistic electrodynamics with travelling waves
}

\author{
Gaetano Fiore ${ }^{1,2, \star}$ \\ ${ }^{1}$ Dip. di Matematica e Applicazioni, Università di Napoli "Federico II", Complesso Universitario MSA, Via Cintia, Napoli, Italy \\ ${ }^{2}$ INFN, Sezione di Napoli, Complesso Universitario M. S. Angelo, Via Cintia, Napoli, Italy
}

\begin{abstract}
We briefly report on our method [23] of simplifying the equations of motion of charged particles in an electromagnetic (EM) field that is the sum of a plane travelling wave and a static part; it is based on changes of the dependent variables and the independent one (light-like coordinate $\xi$ instead of time $t$ ). We sketch its application to a few cases of extreme laser-induced accelerations, both in vacuum and in plane problems at the vacuum-plasma interface, where we are able to reduce the system of the (Lorentz-Maxwell and continuity) partial differential equations into a family of decoupled systems of Hamilton equations in 1 dimension. Since Fourier analysis plays no role, the method can be applied to all kind of travelling waves, ranging from almost monochromatic to socalled "impulses".
\end{abstract}

\section{Introduction and set-up}

The equation of motion of a particle with charge $q$ in external electric and magnetic fields $\mathbf{E}(\mathrm{x}), \mathrm{B}(\mathrm{x})[\mathrm{x} \equiv(c t, \mathbf{x})]$ in its general form is non-autonomous and highly nonlinear:

$$
\begin{aligned}
\dot{\mathbf{p}}(t) & =q \mathbf{E}[c t, \mathbf{x}(t)]+q \boldsymbol{\beta}(t) \wedge \mathbf{B}[c t, \mathbf{x}(t)], \\
\dot{\mathbf{x}}(t) & =\frac{c \mathbf{p}(t)}{\sqrt{m^{2} c^{2}+\mathbf{p}^{2}(t)}} ;
\end{aligned}
$$

here $\beta \equiv \mathbf{v} / c, \mathbf{p} \equiv m \mathbf{v} / \sqrt{1-\beta^{2}}$ is its relativistic momentum.

Usually, (1) is simplified assuming:

1. E, B are constant or vary "slowly" in space/time; or

2. E, B are "small" (so that nonlinear effects in E, B are negligible); or

3. E, B are monochromatic waves, or slow modulations of; or

4. the motion of the particle keeps non-relativistic.

The astonishing developments of Laser technologies (especially Chirped Pulse Amplification [2, 3]) today allow the construction of compact sources of extremely intense (up to $10^{23} \mathrm{~W} / \mathrm{cm}^{2}$ ) coherent EM waves, possibly concentrated in very short laser pulses ( $\gtrsim \mathrm{fs}$ ). Even more intense/short (or cheaper) laser pulses by new technologies (thin film compression [4], etc.) will be soon available. In particular, these lasers can be used for making small particle-accelerators based on Laser Wake Field Acceleration (LWFA) [5] in plasmas. Extreme conditions are present also in several violent astrophysical processes (see

\footnotetext{
^e-mail: gaetano.fiore@na.infn.it
}

e.g. [6] and references therein). In either case the effects are so fast, huge, highly nonlinear, ultra-relativistic that conditions 1-4 are not fulfilled. Alternative simplifying approaches are therefore desirable.

Here we summarize a new approach [23] that is especially fruitful if in the spacetime region $\Omega$ of interest (i.e., where we wish to follow the charged particles' worldlines) E, B can be decomposed into a static part and a plane transverse travelling wave propagating in the $z$ direction:

$$
\begin{aligned}
& \mathbf{E}(\mathrm{x})=\underbrace{\boldsymbol{\epsilon}^{\perp}(c t-z)}_{\text {pump=travelling wave }}+\underbrace{\mathbf{E}_{s}(\mathbf{x}),}_{\text {static }} \\
& \mathbf{B}(\mathrm{x})=\underbrace{\mathbf{k} \wedge \boldsymbol{\epsilon}^{\perp}(c t-z)}_{\text {travelling wave }}+\underbrace{\mathbf{B}_{s}(\mathbf{x}),}_{\text {static }},
\end{aligned}
$$

$\mathbf{x}=x \mathbf{i}+y \mathbf{j}+z \mathbf{k}, \boldsymbol{\epsilon}^{\perp} \perp \mathbf{k}$. We decompose vectors as $\mathbf{u}=\mathbf{u}^{\perp}+u^{z} \mathbf{k}$. We assume only that $\boldsymbol{\epsilon}^{\perp}(\xi)$ is piecewise continuous and

$$
\begin{aligned}
& \text { a) } \boldsymbol{\epsilon}^{\perp} \text { has a compact support }[0, l], \\
& \text { or } \quad \text { ') } \boldsymbol{\epsilon}^{\perp} \in L^{1}(\mathbb{R}), \\
& \Rightarrow \boldsymbol{\alpha}^{\perp}(\xi) \equiv-\int_{-\infty}^{\xi} d y \boldsymbol{\epsilon}^{\perp}(y) \rightarrow 0 \quad \text { as } \xi \rightarrow-\infty ;
\end{aligned}
$$

$\alpha^{\perp}$ is the travelling-wave part of the transverse EM potential $\mathbf{A}^{\perp}$. a) $\Rightarrow \boldsymbol{\alpha}^{\perp}(\xi)=0$ if $\xi \leq 0, \boldsymbol{\alpha}^{\perp}(\xi)=\boldsymbol{\alpha}^{\perp}(l)$ if $\xi \geq l$. We can treat on the same footing all such $\boldsymbol{\epsilon}^{\perp}$, in particular:

1. A modulated monochromatic wave fulfilling (3):

$$
\begin{gathered}
\boldsymbol{\epsilon}^{\perp}(\xi)=\underbrace{\epsilon(\xi)}_{\text {modul. }} \underbrace{\left[\mathbf{i} a_{1} \cos (k \xi+\varphi)+\mathbf{j} a_{2} \sin (k \xi)\right]}_{\text {carrier wave } \boldsymbol{\epsilon}_{o}^{\perp}(\xi)} \\
\Rightarrow-\boldsymbol{\alpha}^{\perp}(\xi)=\frac{\epsilon(\xi)}{k^{2}} \boldsymbol{\epsilon}_{o}^{\perp \prime}(\xi)+O\left(\frac{1}{k^{2}}\right) \simeq \frac{\epsilon(\xi)}{k^{2}} \boldsymbol{\epsilon}_{o}^{\perp \prime}(\xi)
\end{gathered}
$$




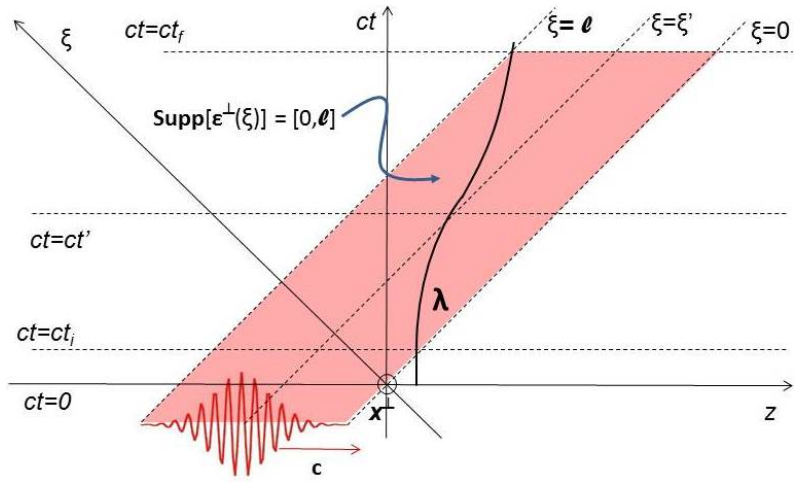

Figure 1. Every worldline $\lambda$ and hyperplane $\xi=\mathrm{C}$ intersect once.

\section{A superposition of waves of type 1 .}

3. An 'impulse' (few cycles, or even a fraction of).

The idea is: as no particle can reach the speed of light $c$, $\tilde{\xi}(t)=c t-z(t)$ is strictly growing, and we can adopt $\xi=c t-z$ as a parameter on the worldline $\lambda$ (see fig. 1) and in the action functional of the particle:

$$
\begin{aligned}
& S(\lambda)=-\int_{\lambda} m c^{2} d \tau+q A(\mathrm{x}) \\
& =-\int_{t_{0}}^{t_{1}} d t \underbrace{\frac{m c^{2}+q u^{\mu} A_{\mu}}{\gamma}}_{L[\mathbf{x}, \mathbf{x}, t]}=-\int_{\xi_{0}}^{\xi_{1}} \frac{d \xi}{c} \underbrace{\frac{m c^{2}+q \hat{u}^{\mu} \hat{A}_{\mu}}{\hat{s}}}_{\mathcal{L}\left[\hat{\mathbf{x}}, \hat{\mathbf{x}}^{\prime}, \xi\right]} ;
\end{aligned}
$$

$\left(u^{\mu}\right)=\left(u^{0}, \mathbf{u}\right) \equiv(\gamma, \gamma \boldsymbol{\beta})$ is the 4-velocity, i.e. the dimensionless version of the 4-momentum. $A(\mathrm{x})=A_{\mu}(\mathrm{x}) d \mathrm{x}^{\mu}=$ $A^{0}(\mathrm{x}) c d t-\mathbf{A}(\mathrm{x}) \cdot d \mathbf{x}$ is the EM potential 1-form, $\mathbf{E}=$ $-\partial_{t} \mathbf{A} / c-\nabla A^{0}, \mathbf{B}=\nabla \wedge \mathbf{A}$ (we use Gauss CGS units), and we denote $\hat{\mathbf{x}}(\xi)=\mathbf{x}(t), \hat{f}(\xi, \hat{\mathbf{x}}) \equiv f(c t, \mathbf{x}), \hat{f}^{\prime} \equiv d \hat{f} / d \xi$ for all functions $f(c t, \mathbf{x})$. Applying Hamilton's principle and the Legendre transform we find simplified Lagrange, and Hamilton equations where the argument of $\boldsymbol{\epsilon}^{\perp}$ is the independent variable $\xi$, rather than the unknown $c t-z(t)$, and the new kinetic momenta are the dependent variables.

\section{General results for one particle}

To parametrize $\lambda$ by $\xi$ we have to replace $d \tau / d t=1 / \gamma=$ $\sqrt{1-\dot{\mathbf{x}}^{2} / c^{2}}$ ( $\tau$ is the particle proper time) by

$$
\frac{1}{\hat{s}} \equiv \frac{d(c \tau)}{d \xi}=\sqrt{1+2 \hat{z}^{\prime}-\hat{\mathbf{x}}^{\perp \prime 2}}>0
$$

From $\mathbf{p}=m d \mathbf{x} / d \tau, \gamma=d t / d \tau$ we find that the $s$-factor $\hat{s}$ is the light-like component $\hat{u}^{-}=\hat{\gamma}-\hat{u}^{z}$ of the 4-velocity $u=$ $\left(u^{0}, \mathbf{u}\right) \equiv(\gamma, \gamma \boldsymbol{\beta})=\left(\frac{p^{0}}{m c^{2}}, \frac{\mathbf{p}}{m c}\right)$ (all these are dimensionless), and $\hat{\mathbf{u}}=\hat{\boldsymbol{s}} \hat{\mathbf{x}}^{\prime} . \hat{\gamma}, \hat{u}^{z}, \hat{\boldsymbol{\beta}}, \hat{\mathbf{x}}^{\prime}$ can be expressed as rational functions of $\hat{\mathbf{u}}^{\perp}, \hat{s}$ :

$$
\begin{array}{ll}
\hat{\gamma}=\frac{1+\hat{\mathbf{u}}^{\perp 2}+\hat{s}^{2}}{2 \hat{s}}, & \hat{u}^{z}=\hat{\gamma}-\hat{s}, \quad \hat{\boldsymbol{\beta}}=\frac{\hat{\mathbf{u}}}{\hat{\gamma}}, \\
\hat{\mathbf{x}}^{\perp \prime}=\frac{\hat{\mathbf{u}}^{\perp}}{\hat{s}}, & \hat{z}^{\prime}=\frac{1+\hat{\mathbf{u}}^{\perp 2}}{2 \hat{s}^{2}}-\frac{1}{2}
\end{array}
$$

By Hamilton's principle, any extremum $\lambda$ of $S$ is the worldline of a possible motion of the particle with initial position $\mathbf{x}_{0}$ at time $t_{0}$ and final position $\mathbf{x}_{1}$ at time $t_{1}$. Hence it fulfills Euler-Lagrange equations in both forms $\frac{d}{d t} \frac{\partial L}{\partial \dot{\mathbf{x}}}=$ $\frac{\partial L}{\partial \mathbf{x}}$ and $\frac{d}{d \xi} \frac{\partial \mathcal{L}}{\partial \hat{\mathbf{x}}^{\prime}}=\frac{\partial \mathcal{L}}{\partial \hat{\mathbf{x}}}$, equivalent to (1). The Legendre transform yields the Hamiltonians $H \equiv \dot{\mathbf{x}} \cdot \frac{\partial L}{\partial \dot{\mathbf{x}}}-L=\gamma m c^{2}+q A^{0}$ and $\hat{H} \equiv \hat{\mathbf{x}}^{\prime} \cdot \frac{\partial \mathcal{L}}{\partial \hat{\mathbf{x}}^{\prime}}-\mathcal{L}=\hat{\gamma} m c^{2}+q \hat{A}^{0} . \quad \hat{H}$ is a rational function of $\hat{\mathbf{x}}, \hat{\boldsymbol{\Pi}} \equiv \frac{\partial \mathcal{L}}{\partial \hat{\mathbf{x}}^{\prime}}$, or, equivalently, of $\hat{s}, \hat{\mathbf{u}}^{\perp}$ :

$$
\begin{array}{r}
\hat{H}(\hat{\mathbf{x}}, \hat{\Pi} ; \xi)=m c^{2} \frac{1+\hat{s}^{2}+\hat{\mathbf{u}}^{\perp 2}}{2 \hat{s}}+q \hat{A}^{0}(\xi, \hat{\mathbf{x}}), \\
\text { where }\left\{\begin{array}{l}
m c^{2} \hat{\mathbf{u}}^{\perp}=\hat{\boldsymbol{\Pi}}^{\perp}-q \hat{\mathbf{A}}^{\perp}(\xi, \hat{\mathbf{x}}), \\
m c^{2} \hat{s}=-\hat{\Pi}^{z}-q \hat{A}^{-}(\xi, \hat{\mathbf{x}}),
\end{array}\right.
\end{array}
$$

while $H(\mathbf{x}, \mathbf{P}, t)=\sqrt{m^{2} c^{4}+(c \mathbf{P}-q \mathbf{A})^{2}}+q A^{0} \quad\left(\mathbf{P} \equiv \frac{\partial L}{\partial \dot{\mathbf{x}}}=\right.$ $\left.\mathbf{p}+\frac{q}{c} \mathbf{A}\right)$ is not. Eq. (1) are also equivalent to the Hamilton equations $\hat{\mathbf{x}}^{\prime}=\frac{\partial \hat{H}}{\partial \hat{\mathbf{\Pi}}}, \hat{\mathbf{\Pi}}^{\prime}=-\frac{\partial \hat{H}}{\partial \hat{\mathbf{x}}}$. All the new equations (in particular these ones) can be also obtained more directly from the old ones by putting a caret on all dynamical variables and replacing $d / d t$ by $(c \hat{s} / \hat{\gamma}) d / d \xi$. Along the solutions $\hat{H}$ gives the particle energy as a function of $\xi$. Under the EM field (2) eqs (1) amount to (9) and

$$
\begin{aligned}
& \hat{\mathbf{u}}^{\perp \prime}=\frac{q}{m c^{2}}\left[\left(1+\hat{z}^{\prime}\right) \hat{\mathbf{E}}_{s}^{\perp}+\left(\hat{\mathbf{x}}^{\prime} \wedge \hat{\mathbf{B}}_{s}\right)^{\perp}+\boldsymbol{\epsilon}^{\perp}(\xi)\right], \\
& \hat{s}^{\prime}=\frac{-q}{m c^{2}}\left[\hat{E}_{s}^{z}-\hat{\mathbf{x}}^{\perp \prime} \cdot \hat{\mathbf{E}}_{s}^{\perp}+\left(\hat{\mathbf{x}}^{\perp \prime} \wedge \hat{\mathbf{B}}_{s}^{\perp}\right)^{z}\right],
\end{aligned}
$$

while the energy gain (normalized to $m c^{2}$ ) is

$$
\mathcal{E} \equiv \frac{\hat{H}\left(\xi_{1}\right)-\hat{H}\left(\xi_{0}\right)}{m c^{2}}=\int_{\xi_{0}}^{\xi_{1}} d \xi q \boldsymbol{\epsilon}^{\perp} \cdot \frac{\hat{\mathbf{u}}^{\perp}}{\hat{s}}
$$

in the interval $\left[\xi_{0}, \xi_{1}\right]$. Once solved (9-12), analytically or numerically, to obtain the solution as a function of $t$ we just need to invert $\hat{t}(\xi)=\xi+\hat{z}(\xi)$ and set $\mathbf{x}(t)=\hat{\mathbf{x}}[\xi(t)]$. If $\mathbf{E}_{s}, \mathbf{B}_{s}=$ const then eq. (12) are solved by

$$
\begin{aligned}
& \hat{\mathbf{u}}^{\perp}=\frac{q}{m c^{2}}\left[\mathbf{K}-\boldsymbol{\alpha}(\xi)+(\xi+\hat{z}) \mathbf{E}_{s}+\hat{\mathbf{x}} \wedge \mathbf{B}_{s}\right]^{\perp}, \\
& \hat{s}=\frac{-q}{m c^{2}}\left[K^{z}+\xi E_{s}^{z}-\hat{\mathbf{x}}^{\perp} \cdot \mathbf{E}_{s}^{\perp}+\left(\hat{\mathbf{x}}^{\perp} \wedge \mathbf{B}_{s}\right)^{z}\right]
\end{aligned}
$$

$\left(K^{j}\right.$ are integration constants) whereby (9) become three $1^{\text {st }}$ order ordinary differential equations (ODE) rational in the unknown $\hat{\mathbf{x}}(\xi)$.

Contrary to (9-12), (1) is a transcendental system, and the unknown $z(t)$ appears in the argument of the rapidly varying functions $\boldsymbol{\epsilon}^{\perp}, \boldsymbol{\alpha}^{\perp}$ in (1) 1 , which now reads:

$$
{ }_{q}^{1} \dot{\mathbf{p}}(t)=\mathbf{E}_{s}+\boldsymbol{\beta} \wedge \mathbf{B}_{s}+\boldsymbol{\epsilon}^{\perp}[c t-z(t)]\left(\boldsymbol{\beta} \mathbf{k}+1-\beta^{z}\right) .
$$

Also determining $\mathcal{E}(t)$ is more complicated.

\subsection{Dynamics under $A^{\mu}=A^{\mu}(t, z)$}

This applies in particular to (2) if $\mathbf{E}_{s}=E_{s}^{z}(z) \mathbf{k}, \mathbf{B}_{s}=\mathbf{B}_{s}^{\perp}(z)$, choosing e.g. $A^{0}=-\int^{z} d \zeta E_{s}^{z}(\zeta), \mathbf{A}^{\perp}=\boldsymbol{\alpha}^{\perp}-\mathbf{k} \wedge \int^{z} d \zeta \mathbf{B}^{\perp}(\zeta)$, $A^{z} \equiv 0$. As $\partial \hat{H} / \partial \hat{\mathbf{x}}^{\perp}=0$, we find $\hat{\mathbf{\Pi}}^{\perp}=q \mathbf{K}^{\perp}=$ const, i.e. the known result $\frac{m c^{2}}{q} \hat{\mathbf{u}}^{\perp}=\mathbf{K}^{\perp}-\hat{\mathbf{A}}^{\perp}(\xi, \hat{z})$. Setting $v:=\hat{\mathbf{u}}^{\perp 2}$ and replacing in $(9)_{2},(\stackrel{q}{12})_{2}$ we obtain

$$
\hat{z}^{\prime}=\frac{1+\hat{v}}{2 \hat{s}^{2}}-\frac{1}{2}, \quad \hat{s}^{\prime}=\frac{-q}{m c^{2}} E_{s}^{z}(\hat{z})-\frac{1}{2 \hat{s}} \frac{\partial \hat{v}}{\partial \hat{z}} .
$$


Once solved the system (15) in the unknowns $\hat{z}(\xi), \hat{s}(\xi)$, the other unknowns are obtained from

$$
\hat{\mathbf{x}}(\xi)=\mathbf{x}_{0}+\hat{\mathbf{Y}}(\xi), \quad \hat{\mathbf{Y}}(\xi) \equiv \int_{\xi_{0}}^{\xi} d y \frac{\hat{\mathbf{u}}(y)}{\hat{s}(y)} .
$$

If in addition $\mathbf{B}_{s} \equiv 0$, then $\mathbf{A}_{s} \equiv 0$ (in the Coulomb gauge), $\hat{\mathbf{u}}^{\perp}(\xi)=\frac{q}{m c^{2}}\left[\mathbf{K}^{\perp}-\boldsymbol{\alpha}^{\perp}(\xi)\right]$ and $\hat{v}=\hat{\mathbf{u}}^{\perp 2}$ are already known. The system (15) to be solved simplifies to

$$
\hat{z}^{\prime}=\frac{1+\hat{v}}{2 \hat{s}^{2}}-\frac{1}{2}, \quad \hat{s}^{\prime}=\frac{-q}{m c^{2}} E_{s}^{z}(\hat{z}) .
$$

Some remarkables properties of the solutions are [23]:

1. Where $\boldsymbol{\epsilon}^{\perp}(\xi)=0$ then $\hat{v}(\xi)=v_{c}=$ const, $\hat{H}$ is conserved, (17) is solved by quadrature.

2. The final transverse momentum is $m c \mathbf{u}^{\perp}\left(\xi_{f}\right)$. If $\epsilon$ of (5) varies slowly and $\mathbf{u}^{\perp}(0)=\mathbf{0}$, then $\mathbf{u}^{\perp}\left(\xi_{f}\right) \simeq 0$.

3. $\hat{s}(\xi)$ is insensitive to fast oscillations of $\boldsymbol{\epsilon}^{\perp}$, contrary to $\mathbf{u}, \gamma, \boldsymbol{\beta}$, which can be reobtained via (8).

\section{Some exact solutions for $\mathbf{B}_{s}, \mathbf{E}_{s}=$ const}

Let $\mathbf{b}^{\perp}+b \mathbf{k} \equiv q \mathbf{B}_{s} / m c^{2}, \mathbf{e}^{\perp} \equiv q \mathbf{E}_{s}^{\perp} / m c^{2}$ (constants), $\boldsymbol{w}(\xi) \equiv$ $q\left[\mathbf{K}-\boldsymbol{\alpha}^{\perp}(\xi)+\xi \mathbf{E}_{s}\right] / m c^{2}$ (all dimensionless); (14) take the more explicit form

$$
\begin{aligned}
& \hat{u}^{x}=\left(e^{x}-b^{y}\right) \hat{z}+b \hat{y}+w^{x}(\xi), \\
& \hat{u}^{y}=\left(e^{y}+b^{x}\right) \hat{z}-b \hat{x}+w^{y}(\xi), \\
& \hat{s}=\left(e^{x}-b^{y}\right) \hat{x}+\left(e^{y}+b^{x}\right) \hat{y}-w^{z}(\xi),
\end{aligned}
$$

For any $E_{s}^{z}, B_{s}^{z}, \mathbf{E}_{s}^{\perp}$, if $\mathbf{B}_{s}^{\perp}=\mathbf{k} \wedge \mathbf{E}_{s}^{\perp}$, setting $\kappa \equiv \frac{q E_{s}^{z}}{m c^{2}}$ we find the following exact solutions (part of them are new):

$$
\begin{aligned}
& (\hat{x}+i \hat{y})(\xi)=(1-\kappa \xi)^{i b / \kappa} \int_{0}^{\xi} d \zeta \frac{\left(w^{x}+i w^{y}\right)(\zeta)}{(1-\kappa \zeta)^{1+i b / \kappa}} \\
& \hat{z}(\xi)=\int_{0}^{\xi} \frac{d \zeta}{2}\left[\frac{1}{(1-\kappa \zeta)^{2}}+\hat{\mathbf{x}}^{\perp^{\prime 2}}(\zeta)-1\right], \quad \hat{s}(\xi)=1-\kappa \xi \\
& \hat{\mathbf{u}}^{\perp}(\xi)=(1-\kappa \xi) \hat{\mathbf{x}}^{\perp \prime}(\xi), \quad \hat{\gamma}(\xi)=1-\kappa \xi+\hat{u}^{z}(\xi) \\
& \hat{u}^{z}(\xi)=\frac{1}{2(1-\kappa \xi)}+(1-\kappa \xi) \frac{\hat{\mathbf{x}}^{\perp \prime 2}(\xi)-1}{2}
\end{aligned}
$$

here we have adopted the initial conditions $\mathbf{x}(0)=\mathbf{0}=\mathbf{u}(0)$. We next analyze a few special cases.

\subsection{Case $\mathbf{E}_{s}=\mathbf{B}_{s}=0$ (zero static fields)}

Then (19) becomes [7, 8]:

$$
\begin{aligned}
& \hat{s} \equiv 1, \quad \hat{\mathbf{u}}^{\perp}=\frac{-q \boldsymbol{\alpha}^{\perp}}{m c^{2}}, \quad \hat{u}^{z}=\frac{\hat{\mathbf{u}}^{\perp 2}}{2}, \quad \hat{\gamma}=1+\hat{u}^{z} \\
& \hat{z}(\xi)=\int_{\xi_{0}}^{\xi} d y \frac{\hat{\mathbf{u}}^{\perp 2}(y)}{2}, \quad \hat{\mathbf{x}}^{\perp}(\xi)=\int_{\xi_{0}}^{\xi} d y \hat{\mathbf{u}}^{\perp}(y) .
\end{aligned}
$$

The solutions (20) induced by two $x$-polarized pulses and the corresponding $e^{-}$trajectories in the $z x$ plane are shown in fig. 2. Note that:
- The maxima of $\gamma, \alpha^{\perp}$ coincide (and approximately also of $\epsilon(\xi)$, if $\epsilon(\xi)$ is slowly varying).

- Since $u^{z} \geq 0$, the $z$-drift is positive-definite. Rescaling $\boldsymbol{\epsilon}^{\perp} \mapsto a \boldsymbol{\epsilon}^{\perp}, \hat{\mathbf{x}}^{\perp}, \hat{\mathbf{u}}^{\perp}$ scale like $a$, whereas $\hat{z}, \hat{u}^{z}$ scale like $a^{2}$ (hence the trajectory goes to a straight line in the limit $a \rightarrow \infty)$. This is due to magnetic force $q \boldsymbol{\beta} \wedge \mathbf{B}$.

- Corollary The final $u$ and energy gain read

$$
\mathbf{u}_{f}^{\perp}=\hat{\mathbf{u}}^{\perp}(\infty), \quad u_{f}^{z}=\mathcal{E}_{f}=\frac{1}{2} \mathbf{u}_{f}^{\perp 2}=\gamma_{f}-1
$$

Both are very small if the pulse modulation $\epsilon$ is slow [extremely small if $\epsilon \in \mathcal{S}(\mathbb{R})$ or $\left.\epsilon \in C_{c}^{\infty}(\mathbb{R})\right]$.

Recall the Lawson-Woodward Theorem [10-13] (an outgrowth of the original Woodward-Lawson Theorem $[14,15])$ : in spite of large energy variations during the interaction, the final energy gain $\mathcal{E}_{f}$ of a charged particle $\mathcal{P}$ interacting with an EM field is zero if:

i) the interaction occurs in $\mathbb{R}^{3}$ vacuum (no boundaries);

ii) $\mathbf{E}_{s}=\mathbf{B}_{s}=\mathbf{0}$ and $\boldsymbol{\epsilon}^{\perp}$ is slowly modulated;

iii) $v^{z} \simeq c$ along the whole acceleration path;

iv) nonlinear (in $\boldsymbol{\epsilon}^{\perp}$ ) effects $q \boldsymbol{\beta} \wedge \mathbf{B}$ are negligible;

v) the power radiated by $\mathcal{P}$ is negligible.

Our Corollary, as Ref. [9], states the same result if we relax iii), iv), but the EM field is a plane travelling wave.

To obtain a non-zero $\mathcal{E}_{f}$ one has to violate some other conditions of the theorem, as e.g. we see in next cases.

3.2 Case $\mathbf{E}_{s}=0, \mathbf{B}_{s}=B_{s}^{z} \mathbf{k}$

Then (19) becomes $\hat{s} \equiv 1$ and

$$
\begin{aligned}
& (\hat{x}+i \hat{y})(\xi)=\int_{0}^{\xi} d \zeta e^{i b(\zeta-\xi)}\left(w^{x}+i w^{y}\right)(\zeta), \hat{\mathbf{u}}^{\perp}=\hat{\mathbf{x}}^{\perp \prime}, \\
& \hat{u}^{z}=\hat{z}^{\prime}=\frac{\hat{\mathbf{u}}^{\perp 2}}{2}=\mathcal{E}=\hat{\gamma}-1, \quad \hat{z}(\xi)=\int_{0}^{\xi} d \zeta \frac{\hat{\mathbf{u}}^{\perp 2}(\zeta)}{2} .
\end{aligned}
$$

(22) reduces to the solution of [16, 17] for monochromatic $\boldsymbol{\epsilon}^{\perp}$. This leads to cyclotron autoresonance if $-b=k=\frac{2 \pi}{\lambda} \gg$ $\frac{1}{l}$ : for circular polarization $w^{x}(\xi)+i w^{y}(\xi) \simeq e^{i k \xi} \mathrm{W}(\xi)$,

$$
(\hat{x}+i \hat{y})(\xi) \simeq i W(\xi) e^{i k \xi}, \quad W(\xi) \equiv \int_{0}^{\xi} d \zeta \mathrm{w}(\zeta)>0
$$

where $\mathrm{w}(\xi) \equiv q \epsilon(\xi) / k m c^{2}$; clearly $W(\xi)$ grows with $\xi$. In particular if $\boldsymbol{\epsilon}^{\perp}(\xi)=\mathbf{0}$ for $\xi \geq l \equiv$, then for such $\xi$

$$
\hat{z}^{\prime}(\xi) \simeq \frac{k^{2}}{2} W^{2}(l) \simeq 2 \mathcal{E}_{f}, \quad \frac{\left|\hat{\mathbf{x}}^{\perp^{\prime}}(\xi)\right|}{\hat{z}^{\prime}(\xi)} \simeq \frac{2}{k W(l)} \ll 1 ;
$$

\subsection{Case $\mathbf{E}_{s}=E_{s}^{z} \mathbf{k}, \mathbf{B}_{s}=\mathbf{0}$}

Then the solution (19) reduces to $\hat{s}(\xi)=1-\kappa \xi$,

$$
(\hat{x}+i \hat{y})(\xi)=\int_{0}^{\xi} d y \frac{\left(w^{x}+i w^{y}\right)(y)}{1-\kappa y}, \quad \hat{z}(\xi)=\int_{0}^{\xi} \frac{d y}{2}\left\{\frac{1+\hat{v}(y)}{[1-\kappa y]^{2}}-1\right\} ;
$$

If $\boldsymbol{\epsilon}^{\perp}$ is slowly modulated the energy gain (13) $\mathcal{E}_{f}$ is negative if $\kappa>0$, positive if $\kappa \leq 0$ and has a unique maximum 

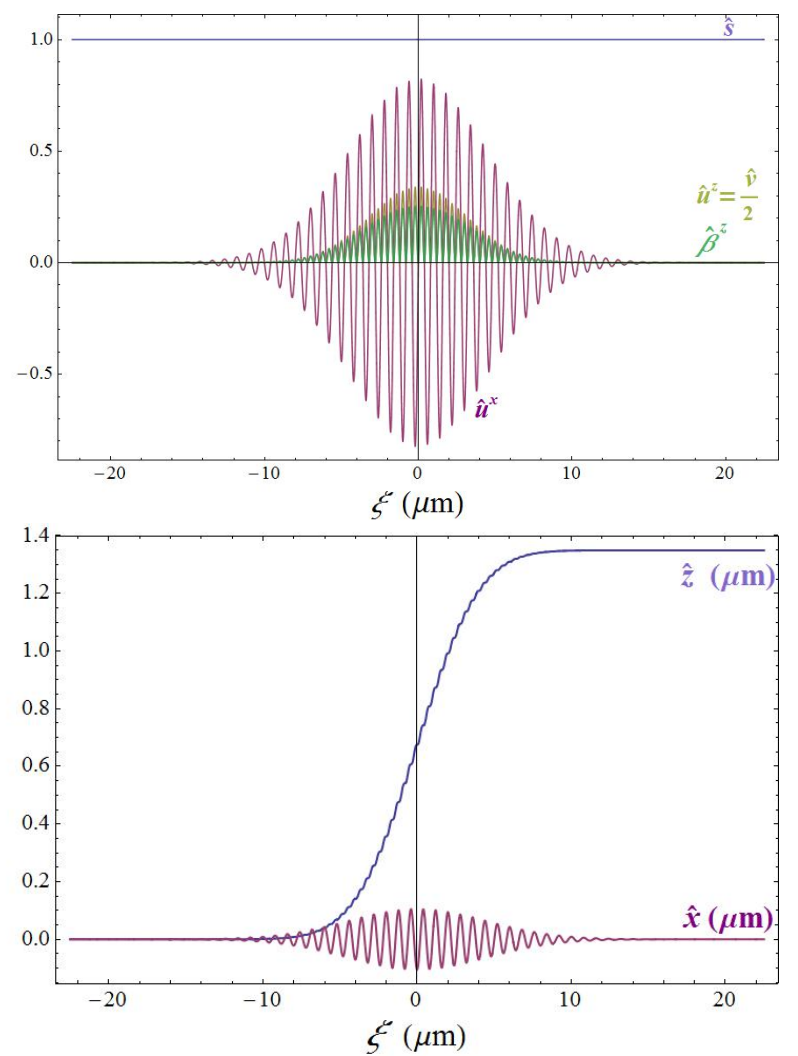
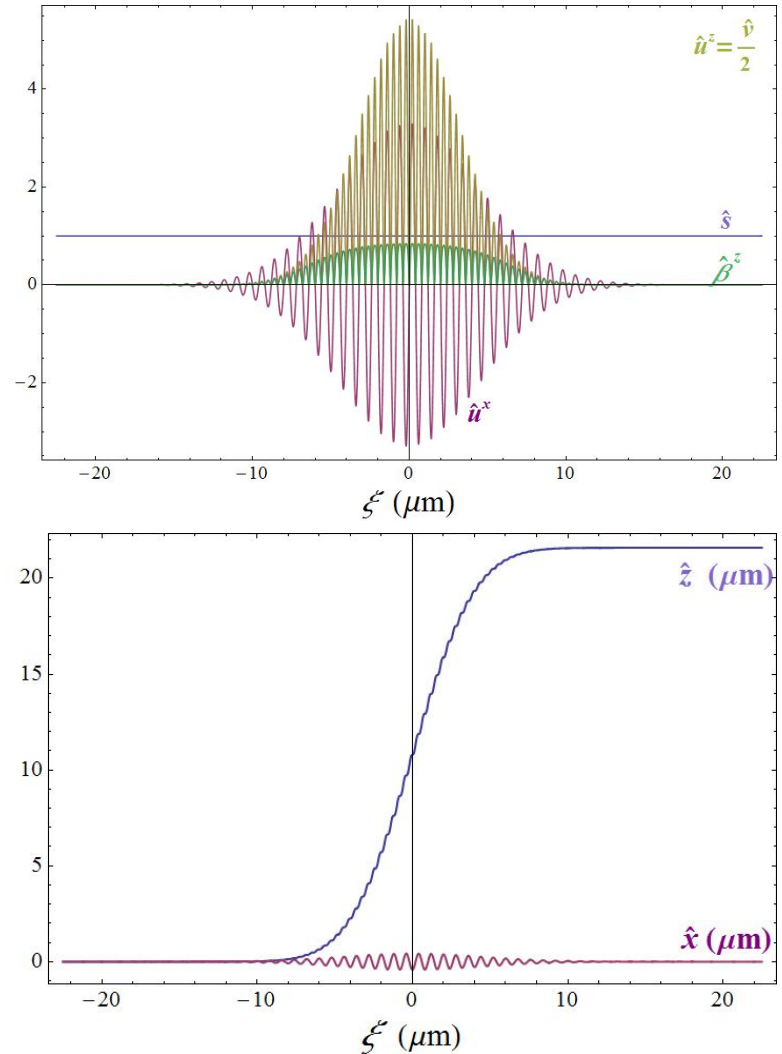
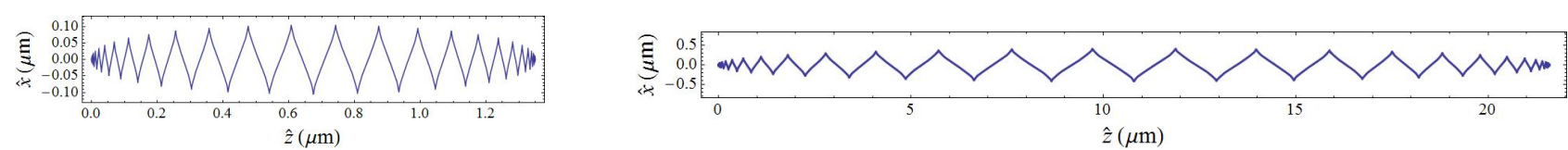

Figure 2. Solutions (20) and $e^{-}$trajectories in the $z x$ plane induced by two $x$-polarized pulses with carrier wavelength $\lambda=.8 \mu \mathrm{m}$, gaussian modulation $\epsilon(\xi)=a \exp \left[-\xi^{2} / 2 \sigma\right], \sigma=20 \mu \mathrm{m}^{2}, e a \lambda / m c^{2}=4,15$ (left, right).

point $\kappa_{M}<0$ if $\epsilon(\xi)$ has a finite support with a unique maximum. Here is an acceleration device based on this solution: at $t=0$ the particle initially lies at rest with $z_{0} \lesssim 0$, just at the left of a metallic grating $G$ contained in the $z=0$ plane and set at zero electric potential; another metallic plate $P$ contained in a plane $z=z_{p}>0$ is set at electric potential $V=V_{p}$. A short laser pulse $\boldsymbol{\epsilon}^{\perp}$ hitting the particle boosts it into the latter region through the ponderomotive force; choosing $q V_{p}>0$ implies $\kappa=-q V_{p} / z_{p} m c^{2}<0$, and a backward longitudinal electric force $q E_{s}^{z}$. If $q V_{p}$ is large enough, then $z(t)$ will reach a maximum smaller than $z_{p}$, then is accelerated backwards and exits the grating with energy $\mathcal{E}_{f}$ and negligible transverse momentum. A large $\mathcal{E}_{f}$ requires extremely large $\left|V_{p}\right|$, far beyond the material breakdown threshold, what prevents its realization as a static field (namely, sparks between $G, P$ would arise and rapidly reduce $\left.\left|V_{p}\right|\right)$. A way out is to make the pulse itself generate such large $\left|E_{s}^{z}\right|$ within a plasma at the right time so as to induce the it slingshot effect, as sketchily explained at the end of next section.

\section{Plane plasma problems}

Assume that the plasma is initially in hydrodynamic conditions with all initial data [velocities, densities $n_{h}$, EM fields of the form (2)] not depending on $\mathbf{x}^{\perp}$. Then also the solutions for $\mathbf{B}, \mathbf{E}, \mathbf{u}_{h}, n_{h}, \Delta \mathbf{x}_{h} \equiv \mathbf{x}_{h}(t, \mathbf{X})-\mathbf{X}$ (displacements) do not depend on $\mathbf{x}^{\perp}$. Here $\mathbf{x}_{h}(t, \mathbf{X})$ is the position at $t$ of the $h$-th fluid material element with initial position $\mathbf{X} \equiv(X, Y, Z) ; \mathbf{X}_{h}(t, \mathbf{x})$ is the inverse (at fixed $\left.t\right)$. More specifically, we consider the impact of an EM plane wave with a pump of the type (3.a) on a cold plasma at equilibrium (figure below); the initial conditions are:

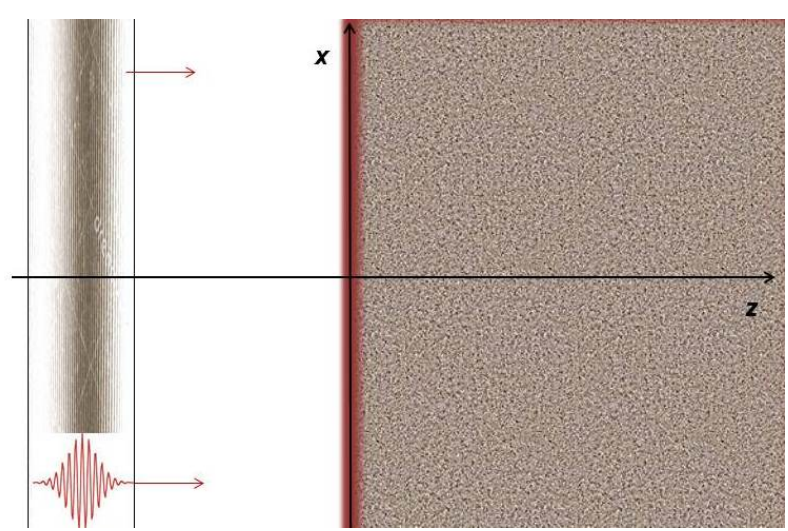




$$
\begin{aligned}
& \mathbf{u}_{h}(0, \mathbf{x})=\mathbf{0}, \quad n_{h}(0, \mathbf{x})=0 \quad \text { if } z \leq 0, \\
& j^{0}(0, \mathbf{x})=\sum_{h} q_{h} n_{h}(0, \mathbf{x}) \equiv 0, \\
& \mathbf{E}(0, \mathbf{x})=\boldsymbol{\epsilon}^{\perp}(-z), \quad \mathbf{B}(0, \mathbf{x})=\mathbf{k} \wedge \boldsymbol{\epsilon}^{\perp}(-z)+\mathbf{B}_{s} .
\end{aligned}
$$

Then Maxwell eq.s $\nabla \cdot \mathbf{E}=4 \pi j^{0}, \partial_{t} E^{z} / c+4 \pi j^{z}=(\nabla \wedge \mathbf{B})^{z}=0$ (the current density is $\mathbf{j}=\sum_{h} q_{h} n_{h} \boldsymbol{\beta}_{h}=\sum_{h} q_{h} n_{h} \frac{\mathbf{u}_{h}}{\gamma_{h}}$ ) imply [8]

$$
E^{z}(t, z)=4 \pi \sum_{h} q_{h} \widetilde{N}_{h}\left[Z_{h}(t, z)\right],
$$

where $\widetilde{N}_{h}(Z) \equiv \int_{0}^{Z} d \zeta n_{h}(0, \zeta)$ : we thus reduce by one the number of unknowns, expressing $E^{z}$ in terms of the (still unknown) longitudinal motion. $\mathbf{A}^{\perp}$ is coupled to the currents through $\square \mathbf{A}^{\perp}=4 \pi \mathbf{j}^{\perp}$ (in the Landau gauges). Including (24) this amounts to the integral equation

$$
\mathbf{A}^{\perp}-\boldsymbol{\alpha}^{\perp}-\frac{\mathbf{B}_{s}}{2} \wedge \mathbf{x}=2 \pi \int d s d \zeta \theta(c t-s-|z-\zeta|) \theta(s) \mathbf{j}^{\perp}\left(\frac{s}{c}, \zeta\right) .
$$

The right-hand side (rhs) is zero for $t \leq 0$, because $t=0$ is the beginning of the laser-plasma interaction. Within short time intervals $\left[0, t^{\prime}\right]$ (to be determined $a$ posteriori) we can approximate $\mathbf{A}^{\perp}(t, z) \simeq \boldsymbol{\alpha}^{\perp}(c t-z)+\frac{\mathbf{B}_{s}}{2} \wedge \mathbf{x}$; we also neglect the motion of ions with respect to that of electrons. Then the Hamilton equations for the electron fluid with 'time' $\xi$ and the initial conditions amount to (9) and

$$
\begin{aligned}
& m c^{2} \hat{s}_{e}^{\prime}(\xi, Z)=4 \pi e^{2}\left[\widetilde{N}\left(\hat{z}_{e}\right)-\widetilde{N}(Z)\right]+e\left(\hat{\mathbf{x}}_{e}^{\perp \prime} \wedge \hat{\mathbf{B}}_{s}^{\perp}\right)^{z}, \\
& m c^{2} \hat{\mathbf{u}}_{e}^{\perp \prime}(\xi, Z)=e \boldsymbol{\alpha}^{\perp \prime}-e\left(\hat{\mathbf{x}}_{e}^{\prime} \wedge \hat{\mathbf{B}}_{s}\right)^{\perp}, \\
& \hat{\mathbf{x}}_{e}(0, \mathbf{X})=\mathbf{X}, \quad \hat{\mathbf{u}}_{e}(0, \mathbf{X})=\mathbf{0} \Rightarrow \hat{s}_{e}(0, \mathbf{X})=1 .
\end{aligned}
$$

this is a family parametrized by $Z$ of decoupled ODEs which can be solved numerically. The approximation on $\mathbf{A}^{-}(t, z)$ is acceptable as long as the so determined motion makes $|\operatorname{rhs}(26)| \ll\left|\boldsymbol{\alpha}^{\perp}+\frac{\mathbf{B}_{s}}{2} \wedge \mathbf{x}\right|$; otherwise rhs(26) determines the first correction to $\mathbf{A}^{\perp}$; and so on.

If $\mathbf{B}_{s}=\mathbf{0}$, again $(27)_{2}$ is solved by $\hat{\mathbf{u}}_{e}^{\perp}(\xi)=e \boldsymbol{\alpha}^{\perp}(\xi) / m c^{2}$, while, setting $v=\hat{\mathbf{u}}^{\perp 2},(9)_{2},(27)_{1}$ take [20] the form of (17)

$$
\Delta \hat{z}_{e}^{\prime}=\frac{1+v}{2 \hat{s}^{2}}-\frac{1}{2}, \quad \hat{s}_{e}^{\prime}=\frac{4 \pi e^{2}}{m c^{2}}\left\{\widetilde{N}\left[\hat{z}_{e}\right]-\widetilde{N}(Z)\right\} .
$$

If $n_{e}(0, \mathbf{X})=n_{0}=$ const for $Z \geq 0$, then as long as $\hat{z}_{e}(\xi, Z)>0$ (29), (28) reduce to the same Cauchy problem for all $Z$ :

$$
\begin{array}{ll}
\Delta^{\prime}=\frac{1+v}{2 s^{2}}-\frac{1}{2}, & s^{\prime}=M \Delta, \\
\Delta(0)=0, & s(0)=1
\end{array}
$$

with $M \equiv \frac{4 \pi e^{2} n_{0}}{m c^{2}}$. In fig. 4 we show the solution if $\boldsymbol{\epsilon}^{\perp}$ is as in fig. 3 and $n_{0}=2 \times 10^{18} \mathrm{~cm}^{-3} ; s(\xi)$ is indeed insensitive to the fast oscillations of $\boldsymbol{\epsilon}^{\perp}$ (see section 2.1). After the pulse is passed it becomes periodic: a plasma travelling-wave of spacial period $\xi_{H} \simeq 49 \mu \mathrm{m}$ follows the pulse. The other unknowns are obtained through (16). Replacing in rhs(26) we find that $\mathbf{A}^{\perp} \simeq \boldsymbol{\alpha}^{\perp}$ is verified at least for $t \leq 5 \xi_{H} / c$

The above results are based on a laser spot size $R=\infty$ (plane wave). When including corrections due to the finite $R$ (based on causality and heuristic estimates), they imply: the impact of a very short and intense laser pulse on the surface of a cold low-density plasma (or gas, ionized

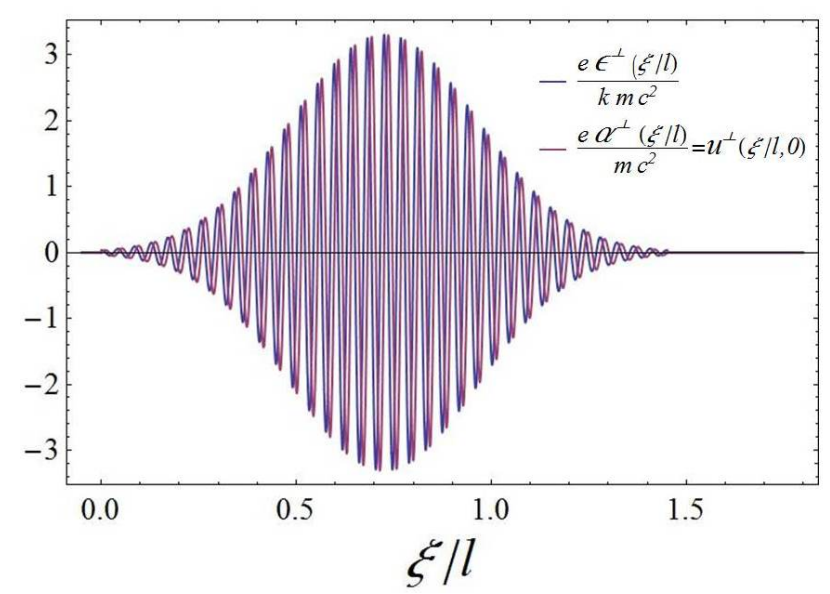

Figure 3. Normalized pump $\boldsymbol{\epsilon}^{\perp}$ with carrier wavelength $\lambda=$ $0.8 \mu \mathrm{m}$, gaussian modulation $\epsilon(\xi)=a \exp \left[-\xi^{2} / 2 \sigma\right], \sigma=20 \mu \mathrm{m}^{2}$, $e a \lambda / m c^{2}=15$, average pulse intensity $10^{19} \mathrm{~W} / \mathrm{cm}^{2}$, linear polarization. $l \simeq 27 \mu \mathrm{m}$ is the length of the interval where the pump amplitude $\epsilon$ overcomes the ionization threshold for the cold gas (here helium) yielding the plasma; under such pulses the thresholds for $1^{\text {st }}$ and $2^{\text {nd }}$ ionization are overcome (i.e. Keldysh parameters become smaller than 1) almost simultaneously [18, 19].

into a plasma by the pulse itself) may induce (for carefully tuned $R$ ), beside a wakefield propagating behind the pulse $[23,24]$, also a backward acceleration and expulsion

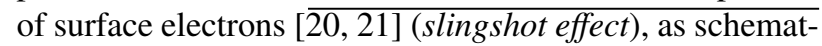
ically depicted in fig. 5. For reviews see also [22].

\section{References}

[1] G. Fiore, arXiv:1607.03482; submitted to J. Phys. A.

[2] D. Strickland, G. Mourou, Opt. Commun. 56 (1985), 219.

[3] G. A. Mourou, T. S. Tajima, V. Bulanov, Rev. Mod. Phys. 78 (2006), 309.

[4] G. Mourou, S. Mironov, E. Khazanov, A. Sergeev, Eur. Phys. J. ST 223 (2014), 1181.

[5] T. Tajima, J.Dawson, Phys.Rev.Lett. 43 (1979), 267.

[6] T. Tajima, K. Nakajima, G. Mourou, Riv. N. Cim. 40 (2017), 34.

[7] L.D. Landau, E.M. Lifshitz, The Classical Theory of Fields, $2^{\text {nd }}$ edition, Pergamon Press, 1962.

[8] G. Fiore, J.Phys.A: M.Th. 47 (2014), 225501

[9] A. L. Troha et al., Phys. Rev. E 60 (1999), 926.

[10] J. D. Lawson, Eur. J. Phys. 5 (1984), 104.

[11] R. B. Palmer, in Frontiers of Particle Beams, Lecture Notes in Physics 296, p. 607, Springer-Verlag, 1988.

[12] R. B. Palmer, AIP Conf. Proc. No. 335 (1995), pp. 90-100; and references therein.

[13] E. Esarey, P. Sprangle, J. Krall Phys. Rev. E 52 (1995), 5443.

[14] P.M. Woodward, J. Inst. Electr. Eng. 93 (1947), 1554.

[15] P. M. Woodward, J. D. Lawson, J. I.E.E. 95, Part III (1948), 363; and references therein. 

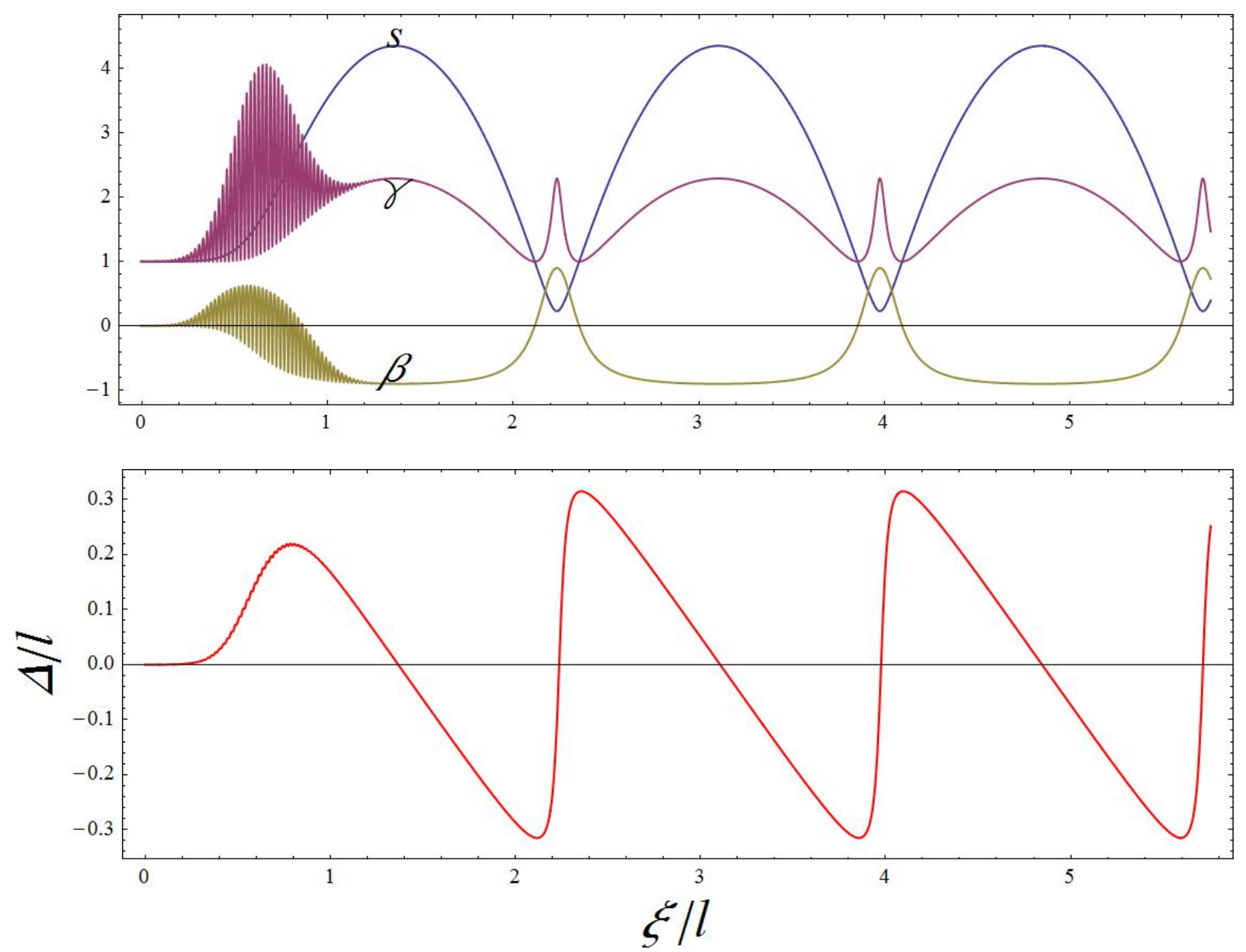

Figure 4. Solution of (29-8) corresponding to the pulse of fig. 3, initial density $\widetilde{n_{e 0}}(Z)=n_{0} \theta(Z), n_{0}=2 \times 10^{18} \mathrm{~cm}^{-3}$.
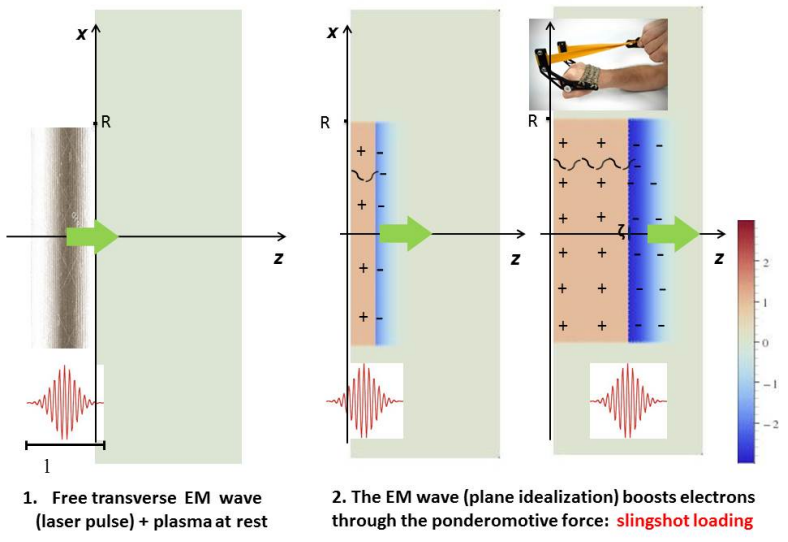

2. The EM wave (plane idealization) boosts electrons through the ponderomotive force: slingshot loading

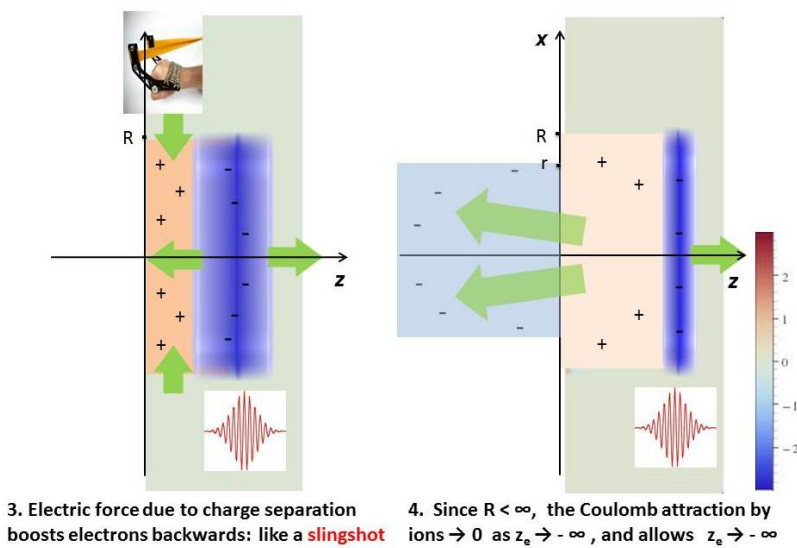

Figure 5. Schematic stages of the slingshot effect.

[16] A.A. Kolomenskii, A.N. Lebedev, Sov. Phys. Dokl. 7745 (1963); Sov. Phys. JETP 17179 (1963).

[17] V. Ya. Davydovskii, Sov. Phys. JETP 16629 (1963).

[18] A. Pukhov, Rep. Prog. Phys. 65 (2002), R1-R55.

[19] D. Jovanović, R. Fedele, F. Tanjia, S. De Nicola, L. A. Gizzi, Eur. Phys. J. D66 (2012), 328.

[20] G. Fiore, S. De Nicola, PRAB 19 (2016), 071302; NIMA 829 (2016), 104.
[21] G. Fiore, R. Fedele, U. de Angelis, Phys. Plasmas 21 (2014), 113105.

[22] G. Fiore, Acta Appl. Math. 132 (2014), 261; Ricerche Mat. 65 (2016), 491-503.

[23] G. Fiore, On the impact of short laser pulses on cold diluted plasmas, in preparation.

[24] G. Fiore, On cold diluted plasmas hit by short laser pulses, submitted to NIMA proceedings. 